\title{
UNA ESPECIE NUEVA DE Eleutherodactylus (AMPHIBIA; ANURA; BRACHYCEPHALIDAE) DE LA AMAZONÍA CENTRAL DEL PERÚ
}

\author{
Victor R. Morales ${ }^{1}$
}

\begin{abstract}
RESUMEN
Se describe una especie nueva de Eleutherodactylus del grupo unistrigatus y del complejo lacrimosus. Esta especie presenta la abertura de las narinas dirigidas hacia arriba en la región nasal, normalmente las especies de anfibios presentan la abertura de las narinas dirigidas hacia un costado. Se distribuye en el bosque tropical lluvioso del Perú.
\end{abstract}

Palabras claves: Amazonía, Anura, Eleutherodactylus, complejo lacrimosus, Perú

\section{SUMMARY}

Herein, we describe a new species of Eleutherodactylus. The new species is placed in the unistrigatus group, lacrimosus assemblage, and can be distinguished from other frogs by having the opening of narines oriented dorsally and not laterally as usual in most anurans. This species is distributed in the Tropical Rain Forest from Perú.

Key words: Amazon, Anura, Eleutherodactylus, lacrimosus assemblage, Perú

Museo de Historia Natural, Universidad Ricardo Palma, Av. Benavides 5440, Santiago de Surco. Apartado Postal 1801, Lima 33, Perú. 


\section{INTRODUCCIÓN}

El genero Eleutherodactylus esta conformado por 491 especies (Frost, 2007) distribuidas en Centro América, Norte América, Sudamérica y las Islas Caribeñas. Para el Perú se reconocen 74 especies (Frost, 2007) organizadas en ocho grupos supra-específicos: conspicillatus, discoidalis, fitzingeri, galdi, nigrovittatus, orestes, sulcatus y unistrigatus. Siendo unistrigatus uno de los grupos con mayor número de especies y dividido en 16 complejos (Lynch y Duellman, 1980). Entre estos complejos está el lacrimosus que fue definido como una unidad fenética por el micro hábitat en común (axilas de las bromelias) de sus especies (Lynch y Ruíz-Carranza, 1985). Recientemente, Guayasamín et al. (2006) resumieron a seis caracteres morfológicos para diagnosticar las especies del complejo lacrimosus y reconocieron 15 especies: Eleutherodactylus apiculatus Lynch y Burrowes 1990; E. aureolineatus Guayasamín, et al. 2006; E. boulengeri Lynch 1981; E. brevifrons Lynch 1981; $E$. bromeliaceus Lynch 1979; E. dorsopictus Rivero y Serna 1988 "1987"; E. eremitus Lynch 1980; E. lacrimosus (Jiménez de la Espada 1875); E. mendax Duellman 1978; E. olivaceus Köhler, Morales, Lötters, Reichle, y Aparicio 1998; E. petersorum Lynch 1991; E. prolixodiscus Lynch 1978; E. schultei Duellman 1990; E. tayrona Lynch y Ruiz-Carranza $1985 ;$ y $E$. zimmermanae Heyer y Hardy 1991. Todos los miembros del complejo lacrimosus, a parte de vivir en las bromelias, presentan una coloración dorsal amarilla, amarillenta-verdosa o verde oliva y también pueden presentar algunos puntos marrones ó diseños en forma de $\mathrm{X}$ ó $\mathrm{W}$.

En este trabajo se describe una especie nueva de Eleutherodactylus de coloración amarillenta, perteneciente al grupo unistrigatus y al complejo lacrimosus. Esta especie ha sido encontrada durante la noche sobre hojas, aproximadamente a 2 metros del suelo.

\section{MATERIAL Y MÉTODOS}

El material tipo fue depositado en el Departamento de Herpetología del Museo de Historia Natural de la Universidad Nacional Mayor de San Marcos (MHNSM).

Los caracteres utilizados para la diagnosis se hicieron siguiendo a Lynch (1979) y Lynch y Duellman (1980). A parte se adicionó un nuevo carácter para el complejo lacrimosus que es la posición de la abertura de las narinas en la región nasal:

Posición de las aberturas de las narinas.- En todas las especies de este complejo la posición de las 
aberturas de la narina se encuentran dirigidas lateralmente en la punta del hocico y en esta especie nueva las aberturas de las narinas se dirigen hacia arriba en la punta del hocico (Fig. 2). Para la definición de las formas del hocico y la terminología de la vocalización se siguió a Heyer et al. (1990). El análisis de la vocalización fue hecho con Canary 1.2.4 (Charif et al. 1995).

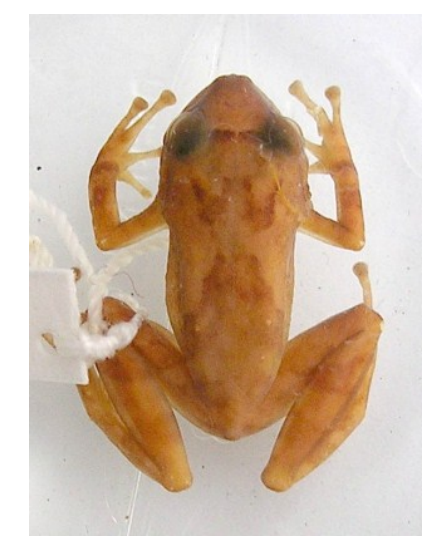

Fig. 1: Patrón dorsal de Eleutherodactylus royi sp. nov. (MHNSM 11187, Holotipo) mostrando el diseño $W$.

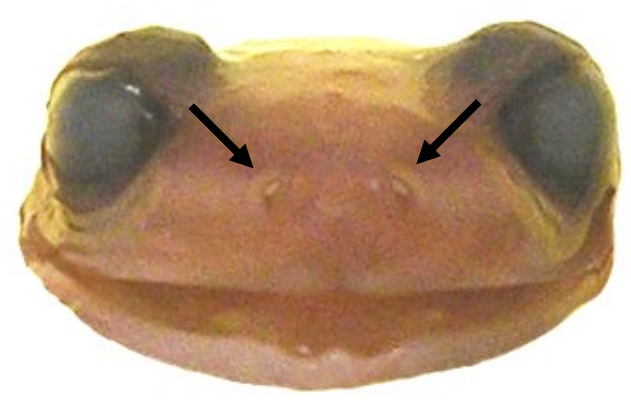

Fig. 2: Vista frontal de la región nasal de Eleutherodactylus royi sp. nov. (MHNSM 11187, Holotipo) mostrando por las flechas) la posición hacia arriba de las narinas. No a escala.
Holotipo.- MHNSM 11187; macho adulto; Bosque Castilla de la Comunidad Nativa 7 de junio (Villa América); $75^{\circ} 14^{\prime} \mathrm{W}, 10^{\circ} 10^{\prime} \mathrm{S}$ aprox.; $330 \mathrm{~m}$ de altitud; Provincia de Huancabamba, Departamento de Pasco, Perú. Colector Víctor R. Morales, (VRM 86149), 30.x.1986.

Paratipo.- MHNSM 11186, mismos datos del holotipo (número de campo VRM 16127).

Diagnosis.- Esta especie es considerada dentro del grupo Eleutherodactylus unistrigatus por la posición de la rama mandibular del nervio trigémino con respecto al músculo aductor mandibular superficial externo y considerándose en la condición "S" (Lynch, 1986) y miembro del complejo lacrimosus por ser habitante de bromelias (Lynch y Ruiz-Carranza 1985). A Eleutherodactylus royi se le reconoce por las siguientes características: 1) Piel del dorso generalmente liso y con escasos tubérculos; ventralmente con gránulos areolados que son tubérculos amplios, circulares y pronunciados; 2) Tímpano redondo $\mathrm{y}$ un tercio de su área superior cubierto por el pliegue timpánico, el diámetro del ojo es $59 \%$ más grande que el diámetro del tímpano; 3 ) Aberturas de las narinas posicionadas hacia arriba (Fig. 2), hocico semiredondo visto de perfil con una leve 
proyección en la punta; visto de arriba es mucronato; 4) Espacio ínter orbital ligeramente mas ancho que el diámetro de los ojos (16\% más), párpados con un tubérculo pequeño; 5) tres dientes vomerianos pequeños; 6) Saco bucal externo en la garganta, subgular; 7) Primer dedo manual más corto que el segundo, disco digital del tercer dedo es el doble de ancho que la penúltima falange; 8) Reborde cutáneo delgado en el lado interno del $2^{\text {do }}$ y $3^{\text {er }}$ dedo manual; 9) Tubérculos articulares pequeños o inconspicuos; 10) Tubérculo en el talón ausente, tres tubérculos tarsales internos pequeños o inconspicuos; 11) Tubérculo metatarsal interno ovalado y el triple de tamaño que el externo que es redondo; 12) Dedos del pie con reborde cutáneo delgado; 13) Dorso amarillo con un diseño marrón claro en forma de "W" en la región escapular y una mancha irregular en la región dorsal del sacro, presenta una línea interorbital blanca brillante y otra línea cantal clara; 14) Longitud hocico-abertura cloacal en ambos machos es de 20.1 $\mathrm{mm}$. No se conoce a la hembra.

Eleutherodactylus royi al parecer es la única especie del complejo lacrimosus que posee la posición de las aberturas narinas hacia arriba y ese carácter la diferencia de todos los miembros del complejo. Por otro lado, E. royi se diferencia de E. lacrimosus por el patrón dorsal amarillento con un diseño en forma de $\mathrm{W}$, mientras que el patrón dorsal en E. lacrimosus es amarillo brillante con algunos puntos marrones y sin diseño dorsal. Comparando a $E$. royi con otros miembros del complejo lacrimosus se observa que difiere de E. mendax por tener los discos digitales $1 / 2$ de ancho que las penúltimas falanges, el dorso y flancos lisos y presentan dientes vomerianos; en E. mendax los discos digitales son $1 / 3$ de ancho que las falanges, el dorso y flancos con gránulos cónicos y no presentan dientes vomerianos (Morales e Icochea, 2000). Eleutherodactylus royi se diferencia de $E$. petersorum [antes E. petersi Lynch, 1996] por la ausencia de una membrana basal sobre el segundo y tercer dedo manual, presentes en E. petersorum; E. royi carece de un pliegue metacarpal desarrollado externo y no presenta numerosos tubérculos supernumerarios en la palma, presentes en $E$. bromeliaceus. Eleutherodactylus royi se diferencia de E zimmermanae por presentar dientes vomerianos, ausentes en E. zimmermanae. Eleutherodactylus royi se diferencia de $E$. acuminatus por la ausencia de una línea lateral oscura que va de la punta del hocico hasta antes de la ingle y por no presentar un pequeño ápice en los discos digitales del tercer dedo manual y del cuarto dedo podal, presentes en E. acuminatus. 
Descripción del holotipo.- Hocico redondeado visto lateralmente con una leve proyección a nivel de las narinas, visto de arriba es subovoide mucronato; canthus rostralis recto; región loreal oblicua y redonda; aberturas de las narinas dirigidas hacia arriba (Fig. 2); tímpano visible casi totalmente, cubierto sólo $1 / 3$ por el pliegue supratimpánico; ojos grandes de diámetro 59\% más que el del tímpano; uno a dos gránulos en la parte posterior del tímpano; tres dientes vomerianos pequeños $\mathrm{y}$ dispuestos oblicuamente a las coanas; lengua granulosa y borde posterior bifurcada; no presenta crestas craneales; saco bucal simple y subgular; dedos de las manos libres y con reborde cutáneo en el lado interno del segundo y tercer dedo; primer dedo manual más corto que el segundo; sin tubérculos supernumerarios en la región palmar; tubérculo palmar bilobulado; pliegue metacarpal externo es remplazado por un pequeño tubérculo; disco del tercer y cuarto dedo anterior el doble de ancho que la penúltima falange; disco del primer dedo anterior menos del doble que la penúltima falange; dedos posteriores libres pero con reborde cutáneo delgado; pliegue tarsal interno es remplazado por tres tubérculos pequeños; región plantar sin tubérculos supernumerarios; tubérculo metatarsal interno ovalado $y$ tres veces más ancho que el externo, que es redondo (Fig. 3). Dorso con pequeños y escasos tubérculos; párpado con un tubérculo pequeño; vientre fuertemente granulado inclusive el muslo.
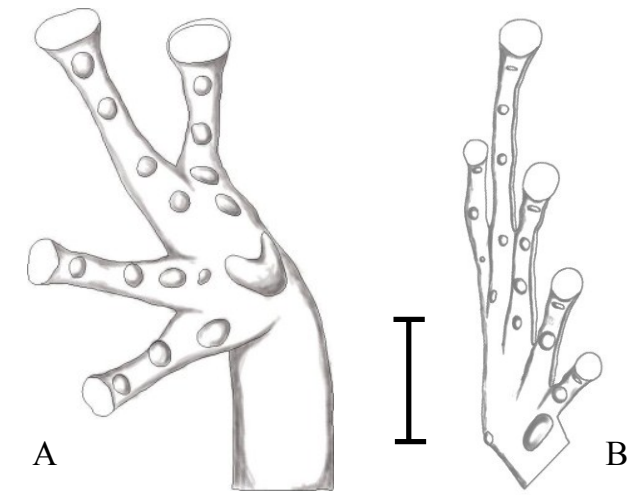

Fig. 3: A) Mano izquierda y B) planta izquierda de Eleutherodactylus royi sp. nov. Holotipo MHNSM 11187. Barra $=1 \mathrm{~mm}$.

Coloración.- Región dorsal marrón claro (amarillo en vivo); una franja marrón oscura entre las órbitas; en la región escapular presenta un diseño marrón oscuro en forma de $\mathrm{W}$; una mancha irregular marrón oscura en la región pélvica; región ventral marrón claro (amarillo brillante en vivo), ojos rojos en vivo.

Medidas $(\mathrm{mm})$ y proporciones del holotipo.- Longitud del hocico a la abertura cloacal (HA), 20.1; largo de la cabeza (LC), 7.7; ancho de la cabeza (AC), 7.5; diámetro del 
tímpano (DT), 1.1; diámetro del ojo (DO), 2.7; distancia interorbital (IO), 3.2; distancia del ojo a la narina (NO), 3.5; largo del muslo (LM), 10.5; largo de la tibia (LT), 10.7; largo del pie, 8.5; LT/HA $=0.53$; $\mathrm{DT} / \mathrm{DO}=0.41 ; \quad \mathrm{IO} / \mathrm{DO}=0.84 ;$ $\mathrm{NO} / \mathrm{AC}=0.47 ; \mathrm{LM} / \mathrm{LT}=0.98$; $\mathrm{AC} / \mathrm{LC}=0.97 ; \mathrm{NO} / \mathrm{HA}=0.17 ;$ $\mathrm{NO} / \mathrm{LC}=0.45$.

Vocalización.- El canto es audible en el bosque durante la noche y consta de un sonido muy corto, como pulsos agudos (Fig. 4). Los pulsos del canto del holotipo, MHNSM 11187, tuvieron una duración de 24.06 a 25.58 mseg $(\chi=24.8, \mathrm{SD}=0.76, \mathrm{n}=3$ pulsos de un solo espécimen). $\mathrm{La}$ frecuencia dominante del canto del holotipo fue de 3270 a $3360 \mathrm{~Hz}(\chi=$ $3300, \mathrm{SD}=0.04, \mathrm{n}=3$ cantos). $\mathrm{El}$ holotipo fue grabado durante la noche (temperatura $=24.2^{\circ} \mathrm{C} ; 21: 00$ horas).

\section{Ecología y distribución.- Ambos} especímenes fueron encontrados sobre vegetación durante la noche; el holotipo se encontraba cantando sobre una hoja $170 \mathrm{~cm}$ del suelo. Se distribuye en las laderas bajas del Bosque Húmedo Tropical o Subandino a $330 \mathrm{~m}$ de altitud en el Centro del Perú.

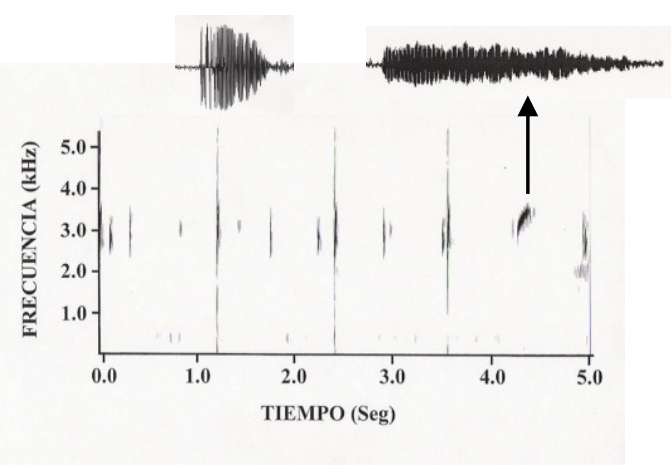

Fig. 4: Audio espectrogramas de las vocalizaciones de Holotipo Eleutherodactylus royi sp. nov. (MHNSM 11187) de la localidad de Bosque Castilla. $24.2^{\circ} \mathrm{C}$; grabado por VRM el 30.oct.1986.

Etimología.- El nombre de la especie es en honor a mi gran amigo Roy W. McDiarmid, profesor, asesor $\mathrm{y}$ colaborador de mis trabajos en la Amazonía Sudamericana.

\section{LITERATURA CITADA}

CHARIF, R.A., MITCHELL, S. \& CLARK, C.W. 1995. Canary 1.2 User's Manual. Cornell Lab. Ornitology, Ithaca, N.Y.

FROST, D. R. 2007. Amphibian Species of the World: an Online Reference. Version 5.0 (1 February, 2007). Electronic Database acessible at http://research.amnh.org/ herpetology/amphibia/index.php.

American Museum of Natural History, New York, USA. 
HEYER, W.R. \& HARDY, L.M. 1991. A new species of frog of the Eleutherodactylus lacrimosus assembly from Amazonia, South America (Amphibia: Anura: Leptodactylidae). Proceedings of the Biological Society of Washington. 104: 436-447.

HEYER, W.R., RAND, A.S., DA CRUZ, C.A.G., PEIXOTO, O.L,. \& NELSON, C.E. 1990. Frogs of Boracéia. Arquivos de Zoologia. 31: 231-410.

LYNCH, J.D. 1976. The species groups of the South American frogs of the genus Eleutherodactylus (Leptodactylidae). Occasional Papers of the Museum of Natural History the University of Kansas. 61: 1-24.

LYNCH, J.D. 1979. Leptodactylid frogs of the genus Eleutherodactylus from the Andes of southern Ecuador. University of Kansas Museum of Natural History Miscellaneous Publication. 61: 162.

LYNCH, J.D. 1986. The definition of the Middle American clade of Eleutherodactylus based on jaw musculature (Amphibia: Leptodactylidae). Herpetologica. 42: 248-258.

LYNCH, J.D. (1996): Replacement names for three homonyms in the genus Eleutherodactylus (Anura: Leptodactylidae). Journal of Herpetology, 30: 278-280.

LYNCH, J.D. \& DUELLMAN, W.E. 1980. The Eleutherodactylus of the amazonian slopes of the Ecuadorian Andes (Anura: Leptodactylidae). University of Kansas Museum of Natural History Miscellaneous Publication. 69: 1-86.

LYNCH, J. D. \& P. M. RUÍZCARRANZA. 1985. A synopsis of the frogs of the genus Eleutherodactylus from the Sierra Nevada de Santa Marta, Colombia. Occasional Papers of the Museum of Zoology, University of Michigan. 711: 1-59.

MORALES, V.R. 1995. Checklist and taxonomic bibliography of the Amphibians from Peru. Smithsonian Herpetological Information Service. 107: 1-20.

MORALES, V.R. \& Icochea, J. 2000. Review of the type material of Eleutherodactylus mendax and a new record of Eleutherodactylus bromeliaceus from Peru. Journal of Herpetology. 34: 158-160.

\section{AGRADECIMIENTOS}

W. Ron Heyer del Smithsonian Institution, Jesús H. Córdova y Javier Icochea del Museo de Historia Natural de la Universidad Nacional Mayor de San Marcos, Perú por el préstamo del material. Al programa Russell E. Train para la educación de la Naturaleza de la WWF (World Wildlfie Fund) por la ayuda en mis investigaciones. 